\title{
Role of alginate in infection with mucoid Pseudomonas aeruginosa in cystic fibrosis
}

\author{
Svend Stenvang Pedersen, Niels Høiby, Frank Espersen, Christian Koch
}

\begin{abstract}
Background Chronic bronchopulmonary infection with mucoid, alginate producing Pseudomonas aeruginosa occurs characteristically in patients with cystic fibrosis. Alginate may be a virulence factor for $P$ aeruginosa infection in such patients.
\end{abstract}

Methods Forced vital capacity (FVC), nutritional state and the antibody response to $\boldsymbol{P}$ aeruginosa were determined at regular intervals from three years before chronic $P$ aeruginosa infection to 10 years afterwards in 73 patients with cystic fibrosis. All patients were treated intensively with antipseudomonal chemotherapy during the study period. Results FVC was reduced in all patients who subsequently developed $P$ aeruginosa infection before they acquired the infection, indicating significant pre-existing lung damage when compared with patients who remained free of $P$ aeruginosa. Lung function and nutritional state remained unchanged after 10 years of infection, except in the patients who died of $P$ aeruginosa lung infection. The FVC and height and weight of patients infected with nonmucoid strains of $P$ aeruginosa were similar to those of uninfected patients. Patients infected with mucoid strains had poorer lung function and nutritional state for the first five years after infection compared with patients with nonmucoid strains. Such infection was also associated with greater IgG and IgA antibody responses to $P$ aeruginosa standard antigen compared with nonmucoid infection. Concentrations of antibody to alginate were similar in patients with non-mucoid and mucoid infection. Noticeably increased concentrations of IgA antibodies to $P$ aeruginosa standard antigen were observed early after the onset of infection in patients who subsequently died.

Conclusion Alginate producing $P$ aeruginosa infection is associated with a hyperimmune response and poor clinical condition, suggesting that alginate production is a virulence factor in such infections in patients with cystic fibrosis.

Mucoid Pseudomonas aeruginosa is isolated from most patients with cystic fibrosis, in whom it causes a chronic, incurable lung infection. Bronchopulmonary disease is the cause of death in $95 \%$ of patients with cystic fibrosis, ${ }^{12}$ and mucoid $P$ aeruginosa was cultured from $94 \%$ of Danish patients with cystic fibrosis at death. ${ }^{3}$ Typically the airways are colonised initially with non-mucoid strains of $P$ aeruginos $a^{45}$ and after a variable period of colonisation, usually one to two years, mucoid strains emerge that produce large amounts of the extracellular polysaccharide alginate. Compared with non-mucoid strains, infection with mucoid $P$ aeruginosa is associated with a poorer clinical state, ${ }^{6}$ lower pulmonary function, ${ }^{78}$ and greater risk of death. ${ }^{7}$

Infection with mucoid strains was also associated with an increased humoral immune response to $P$ aeruginosa..$^{510}$ An increased antibody response is associated with poorer pulmonary function ${ }^{711}$ and the lung damage in cystic fibrosis is mediated by immune complexes. $^{1213}$ This suggests that alginate is a virulence factor in chronic $P$ aeruginosa pulmonary infection in cystic fibrosis. ${ }^{14}$

Our objective was to test the hypothesis that alginate producing, mucoid $P$ aeruginosa is more virulent than non-mucoid strains. We studied 73 patients with cystic fibrosis classified according to their sputum bacteriology longitudinally and determined their antibody responses to $P$ aeruginosa alginate and somatic antigens, lung function, and nutritional state for three years before and for up to 10 years after the onset of chronic $P$ aeruginosa lung infection.

\section{Patients and methods}

The diagnosis of cystic fibrosis was based on repeatedly raised electrolyte concentrations in sweat and characteristic clinical features. The criteria for entry into the study were that the onset of chronic $P$ aeruginosa infection was after 1975, that the patient had been followed up monthly at the Danish Cystic Fibrosis Centre at Rigshospitalet before the onset of infection, and that sputum bacteriology had been recorded for at least eight months of each year. Data were recorded prospectively and included forced vital capacity (FVC), height, weight, and sputum bacteriology. Since 1976 all patients with chronic $P$ aeruginosa infection have been admitted to the centre every three months for intravenous courses of antipseudonomas chemotherapy. ${ }^{15}$

\section{BACTERIOLOGY}

The onset of chronic $P$ aeruginosa infection was defined as the time when $P$ aeruginos $a$ had been grown in consecutive monthly sputum cultures over six months. Sputum was obtained by

\author{
Reprint requests to: \\ Department of Clinical \\ Microbiology \\ Accepted 10 October 1991
}


expectoration or by endolaryngeal aspiration in patients unable to expectorate. The samples were cultured on standard laboratory media and $P$ aeruginosa identified by standard tests. ${ }^{16}$ The mucoid phenotype was identified visually by the appearance of colonies having a typical morphology with an abundance of watery and viscous slime. Strains not showing these characteristics were termed non-mucoid. Patients having both mucoid and non-mucoid colonies in the same sample (a common finding) were classified as harbouring mucoid strains. Strains were defined as virulent when they were associated with a raised antibody response and poor lung function.

\section{CLASSIFICATION OF PATIENTS}

The bacteriological records of all patients with chronic $P$ aeruginosa infection attending the centre at the beginning of 1988 were examined and patients to be included in the study were identified by the above criteria. Some patients did not satisfy these criteria because their onset of infection was before 1976, they had been transferred to the centre after the onset of $P$ aeruginosa infection, or they had not attended regularly. Patients were categorised according to their bacteriological results.

(1) Patients with classic bacteriology had non-mucoid strains initially and intermittently for about six months followed by a period of one to two years of continuous isolation of nonmucoid strains. The mucoid strains started to appear at this point and were cultured continuously thereafter. The median age at onset of infection was 10 years (range 1-16 years) for the 23 patients in this group and the median duration of infection at the time of the study was 10 years (range 6-13 years). Twenty one patients were available for analysis at the final observation (see below).

(2) Patients with non-mucoid bacteriology were chronically infected with non-mucoid $P$ aeruginosa with no mucoid strains isolated from any sputum sample $(n=14)$. The median age at onset of infection was 9 years (range 1-13 years) and the median duration of infection 10 years (range 5-12 years). Nine patients were assessed at the last observation.

(3) Patients with mucoid bacteriology had mucoid colonies at the first isolation of $P$ aeruginosa and remained chronically infected with mucoid strains $(n=9)$. Their median age at onset of infection was 12 years (range 0-31 years) and the median duration of infection nine years (range 4-13 years). Six patients were assessed at the final observation.

(4) Non-colonised patients with cystic fibrosis from whom $P$ aeruginosa had never been isolated were used as a control group $(n=15)$. They were matched for age with the infected patients, and at the end of the study their median age was 19 years (range 12-32 years). At the last observation 13 patients were assessed.

(5) Patients with-fatal $P$ aeruginosa infection were included $(n=12)$. The onset of infection was after 1976 and the median age at onset was 12.5 years (range $0-22$ years). The duration of infection from onset to death was $7 \cdot 2$ years
(2.1-11.5 years). All patients were infected with mucoid strains at the time of death and in four the initial strain was mucoid. The rest showed the same pattern as patients with classic bacteriology. Six patients remained at the final observation for assessment.

\section{CLINICAL MEASUREMENTS}

Clinical assessments were made and serum obtained from each patient every year for three years before the onset of infection; every six months for the first two years after onset; every year from two to five years after onset; and every two years from five years after the onset of infection. FVC was measured by spirometry as the best of three measurements at each clinic visit. A mean value of FVC at each time was expressed as a percentage of predicted values for sex and height. ${ }^{17}$ Because the onset of infection usually takes place in childhood around the age of 10 years paediatric predictions were used. For the sake of comparison the same predictions were used for patients aged 18 years or older.

Height and weight were measured on standard clinic scales at each visit; the mean of the recordings during an interval were expressed as the percentage deviation from the 50th centile of predicted values for Danish children. ${ }^{18}$ For patients older than 18 years the predicted value at age 18 years was used.

\section{TESTS ON SERUM}

Serum samples were stored at $-20^{\circ} \mathrm{C}$ until analysis. All samples were analysed for precipitating antibodies against $P$ aeruginosa, Staphylococcus aureus, and Haemophilus influen$z a e$ by crossed immunoelectrophoresis. ${ }^{19}$ To determine specific antipseudomonal antibody activity the serum sample with the highest number of precipitating bands in a given study interval was selected.

\section{ANTIGENS}

$P$ aeruginosa alginate was isolated and purified from mucoid $P$ aeruginosa..$^{20}$ The alginate did not cross react with antibodies against lipopolysaccharide or protein antigens of $P$ aeruginosa. A mixture of three serologically cross reacting alginates was used. ${ }^{20}$

$P$ aeruginosa standard antigen Water soluble antigens were produced from each of 17 serotypes of the international antigenic typing scheme of $P$ aeruginosa $;{ }^{19}$ equal volumes of each were mixed and designated standard antigen.

\section{ENZYME LINKED IMMUNOSORBENT ASSAYS} (ELISAS)

The antibody response was measured by four ELISAs that detected IgG and IgA antibodies specific for the two different $P$ aeruginosa antigens. All samples from a single patient were run in triplicate on the same assay plate, which also included an internal standard. Details of the assays have been published. ${ }^{1121}$ All reagent volumes were $100 \mu \mathrm{l}$.

Alginate ELISA Plates (MicroWell, Nunc, Tåstrup, Denmark) (96 wells) were coated with $10 \mu \mathrm{g} / \mathrm{ml}$ purified $P$ aeruginosa alginate in phos-

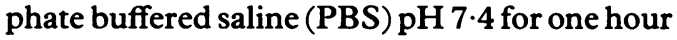


at $35^{\circ} \mathrm{C}$ and residual binding sites were blocked with $0 \cdot 1 \%$ Tween 20 (Polysorbate, Sigma, St Louis, Missouri) in PBS for one hour. IgG antibodies were detected in serum samples diluted 1:4000 in PBS-Tween that were incubated in the wells for one hour at $35^{\circ} \mathrm{C}$. After extensive washings peroxidase conjugated goat-antihuman IgG ( $\gamma$ chain, Tago, Burlingame, California) diluted 1:10000 in PBS-Tween was added for one hour at $35^{\circ} \mathrm{C}$. IgA antibodies to alginate were detected by incubating serum diluted 1:125 for one hour followed by reaction with peroxidase conjugated rabbit-antihuman $\operatorname{Ig} A$ diluted 1:1000 ( $\alpha$ chain, Dako, Glostrup, Denmark).

Standard antigen ELISA Standard antigen $(2 \cdot 2 \mu \mathrm{g} /$ well $)$ was coated on MicroWell plates for IgG antibodies or Maxisorb plates (Nunc, Denmark) for IgA antibodies for one hour at $35^{\circ} \mathrm{C}$. Blocking, washing, and dilution was carried out with PBS-Tween. Serum was diluted 1:4000, the peroxidase conjugated antiIgG (Tago) 1:7500, and anti-IgA (Dako) $1: 1000$. Visualisation of the antigen-antibody reaction was similar for all four ELISAs by adding sodium citrate $(\mathrm{pH} 5 \cdot 0,0 \cdot 1 \mathrm{M})$ containing 1,2 phenylenediamide-dihydrochloride (3.7 mM, Dako) and hydrogen peroxide $(6.5 \mathrm{mM})$ for one hour at room temperature before the reaction was stopped by the addition of $1 \mathrm{M}$ sulphuric acid. Optical density was measured by an automatic ELISA reader (BioRad, Model 3550, Richmond, California) programmed to calculate the antibody response in ELISA units by dividing the mean optical density of the sample by the mean optical density of the internal standard.

Although the assays were optimised to yield an optical density less than 3 (the upper limit of the automatic reader) the optical density of some samples exceeded this and these were further diluted 1:40000 and the assays repeated. The results were multiplied by a dilution factor $(6.8$ for IgG against standard antigen, 4.0 for IgA against standard antigen and 4.0 for IgG against alginate) determined by the linear regression slope for 10 samples analysed in six doubling dilutions. Measurement of IgA antibodies against alginate never exceeded the range so further dilutions were unnecessary.

The performance characteristics of the assays have been described previously. ${ }^{21}$ The within plate variation ranged from $9 \%$ to $19 \%$, the plate to plate variation from $11 \%$ to $16 \%$, and the day to day variation from $13 \%$ to $19 \%$.

\section{ANALYSIS}

For description of distributions and comparison between groups non-parametric analyses were used. For unpaired data the MannWhitney $U$ test was used and for paired data the Wilcoxon signed rank statistic. The level of significance was $5 \%$ for a two tailed comparison.

\section{Results}

There was no difference in the median age at onset of $P$ aeruginosa infection among the four categories of infected patients, or between the infected patients and those from whom colonies were not isolated.

\section{PATIENTS WITHOUT COLONISATION}

Patients with no growth of $P$ aeruginosa had either no or a very low antibody response to $P$ aeruginosa antigens throughout the observation period (figure 1, A-D) when compared with the infected patients $(p<0.0001)$. The FVC was about $90 \%$ of predicted values and did not change during the observation period of 13 years (figure 1, E). Height and weight ranged from $92 \%$ to $100 \%$ and from $98 \%$ to $101 \%$ of the median for healthy Danish children (data not shown). The weight for height median value fluctuated around $100 \%$ of predicted values (figure $1, \mathrm{~F}$ ).

\section{COMPARISON OF PATIENTS WITH MUCOID AND} NON-MUCOID INFECTION

Before infection antibody concentrations for $P$ aeruginosa did not differ between patients who subsequently developed mucoid infection and those who developed non-mucoid infection (figure 1, A-D). Before sputum culture became positive these patients had significantly higher IgA antibody concentrations for $P$ aeruginosa than did those without colonisation. This difference was seen for IgA antibodies to alginate for a period of one year (figure 1, A) and for standard antigen for three years before infection (figure $1, B$ ). Infection with non-mucoid $P$ aeruginosa was associated with an antibody response to alginate that did not differ from that of infection with mucoid strains (figure $1, \mathrm{~A}$ and C). Patients with mucoid infection had a greater IgA response to standard antigen from 1 to 7 years after infection and a greater IgG antibody response from 0 to 5 years than did the patients with non-mucoid infection (figure 1, B and D). Similar differences occurred with precipitating antibodies (data not shown).

Before infection patients who subsequently acquired infection with mucoid strains had a significantly lower FVC than patients with nonmucoid infection (figure 1E). Both groups had significantly poorer FVC than the non-colonised controls (figure 1E). Patients infected with a mucoid organism showed a gradual decline in FVC during the preinfection period, reaching a nadir of $50 \%$ of predicted values a year after the onset of infection. The FVC then improved slowly and five years after the onset of infection had reached preinfection values, where it remained for the rest of the observation period. There was no difference in FVC in this group at the beginning and end of the observation period. The FVC in patients infected with nonmucoid strains had started to improve before the infection became established. Over the 10 years of infection with non-mucoid strains there was no difference in FVC in this group compared with non-colonised patients (figure 1E) whereas the FVC in patients with mucoid infection was significantly worse than that of patients with non-mucoid infection during the five years after the onset of infection (figure 1, E). 
The height and weight of patients with nonmucoid infection was similar to predicted values (data not shown) and the corresponding weight for height was around $100 \%$ of predicted (figure $1 \mathrm{~F}$ ). The nutritional state of patients with non-mucoid infection did not differ from that of non-colonised patients whereas mucoid infection was associated with a significantly lower weight (approximately $85 \%$ of predicted values) for the first five years of infection when compared with patients with non-mucoid infection. The height of patients with mucoid infection (range 95-98\%) did not differ from that of the other groups of patients (data not shown); the weight for height was significantly lower for the first five years of mucoid infection (figure $1 \mathrm{~F}$ ).

\section{COMPARISON OF PATIENTS WITH CLASSIC} BACTERIOLOGY AND MUCOID INFECTION

The course of the antibody response and clinical measurements in 23 patients with the classic pattern of bacteriology are shown in figure 2 (AF). The IgA antibody response to alginate did not differ significantly, except that it was lower from two years before the onset of infection to

Figure 1 Comparison of changes over 14 years in patients with cystic fibrosis with mucoid and nonmucoid infection and noncolonised patients. The antibody responses to $P$ aeruginosa alginate ( $A$ and $C$ ) and to $P$ aeruginosa standard antigen $(B$ and $D)$, forced vital capacity $(E)$, and weight for height $(F)$ are shown. All values shown are medians. Closed circles with solid lines represent patients infected with mucoid $P$ aeruginosa $(n=9)$, open squares and dashed lines patients infected with non-mucoid $P$ aeruginosa $(n=14)$, and closed squares patients not colonised with $P$ aeruginosa $(n=15)$.

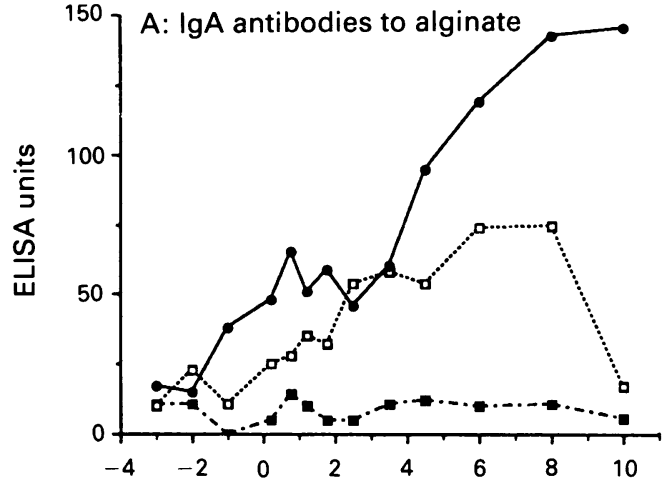

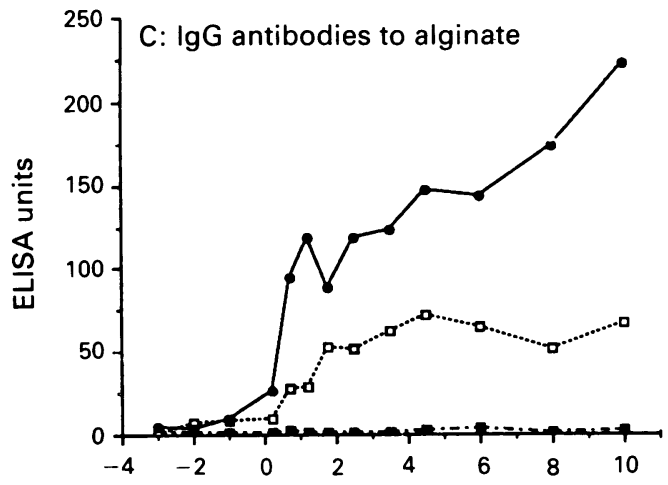
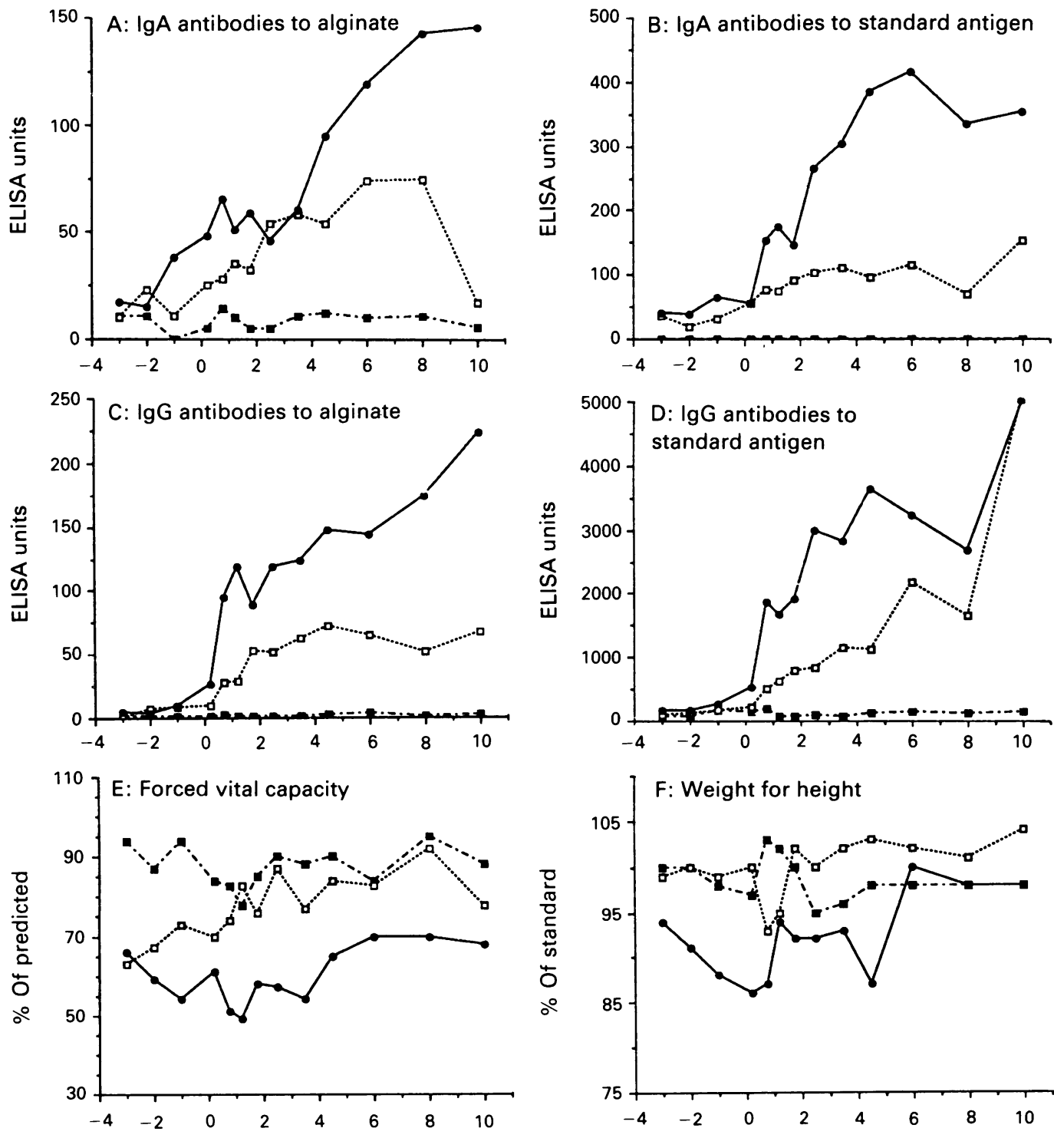

three years after (figure 2, A). The $\operatorname{IgA}$ antibodies to standard antigen were lower in this group of patients than in those with mucoid infection throughout the whole observation period, though this was only significant during the early stages of infection $(0-3$ years) (figure 2, B). The IgG antibodies to standard antigen or alginate were significantly lower only during the first year after the onset of infection and overlapped in the later stages of chronic infection (figure 2, C and D). Precipitating antibodies followed the same trend (data not shown), being significantly lower in patients with classic bacteriology for the first 18 months of infection.

The opposite pattern was observed when the antibody responses in patients with classic bacteriology were compared with those in patients with non-mucoid infection. In the early stages of infection ( $0-3$ years) no difference was noted, after which the response was significantly greater in patients with classic bacteriology. No differences in clinical measures were observed.

Before and at the onset of chronic $P$ aeruginosa infection the patients with classic bac-
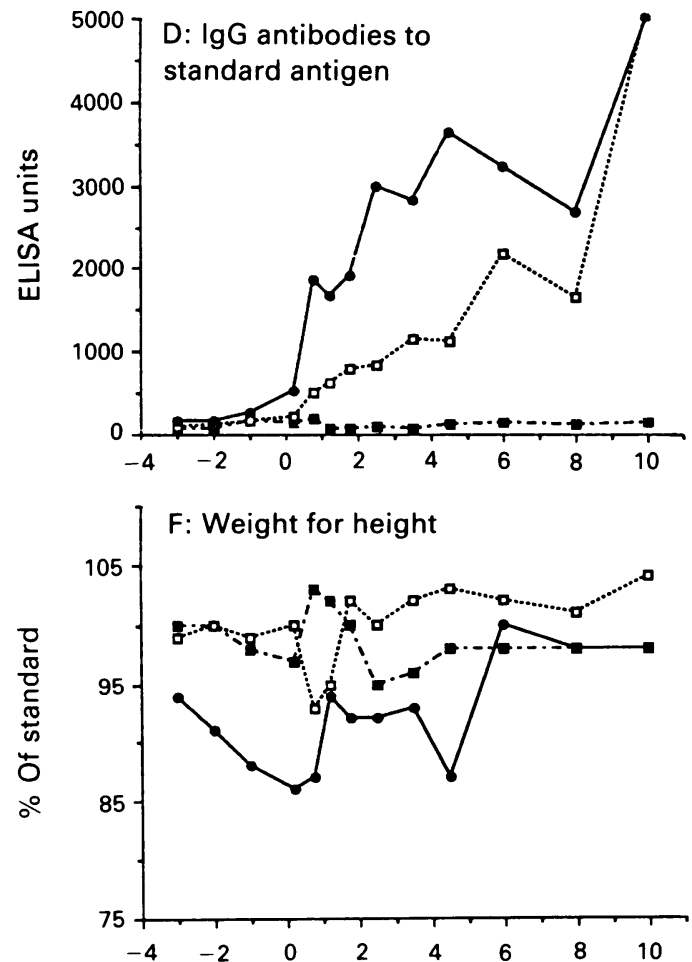

Year after onset of $P$ aeruginosa infection 
teriology had a median FVC of about $70 \%$ of predicted values (figure $2, \mathrm{E}$ ), which was significantly less than that of patients without colonisation but similar to that in patients with mucoid or non-mucoid infection. In the patients with classic bacteriology lung function was unchanged over the 10 years of infection. For one year before and up to three years after acquisition of $P$ aeruginosa lung function was significantly better than in those with mucoid infection (figure 2, E). The lung function in patients with mucoid infection then improved and for the rest of the observation period no difference was found. The weight for height did not differ between patients with mucoid and classic infection (figure $2, F$ ).

\section{LONGITUDINAL STUDY OF PATIENTS WHO DIED FROM $P$ AERUGINOSA INFECTION}

Patients who died from $P$ aeruginosa infection had a similar IgA and IgG antibody response to $P$ aeruginosa to that of those infected with mucoid strains (figure 2, A-D). When compared with patients with classic bacteriology the patients who died had significantly greater
IgA antibody responses to standard antigen and alginate during the whole period of observation (figure 2, A and B). The IgG antibody responses were not different (figure 2, C and D) although precipitating antibodies were significantly greater for the first five years of infection (data not shown).

Before infection the patients who died had similar FVC values to patients with mucoid, non-mucoid, and classic bacteriology but significantly lower values than patients without colonisation. After one year of infection patients with classic or non-mucoid bacteriology had a significantly higher FVC; after four years after the onset of infection the mucoid group had a higher FVC than those who died (figure 2, E).

From the start of infection those who subsequently died had a significantly lower body weight than patients with classic bacteriology. No difference in height was observed (data not shown). They did not differ from patients with mucoid infection until the infection had persisted for five years, after which the weight for height was significantly lower (figure $2, \mathrm{~F}$ ).
Figure 2 Comparison of changes in patients with cystic fibrosis with classic bacteriology with changes in patients who died and in those who had mucoid $P$ aeruginosa infection. The antibody responses to $P$ aeruginosa alginate ( $A$ and $C$ ) and to $P$ aeruginosa standard antigen ( $B$ and $D$ ), forced vital capacity $(E)$, and weight for height $(F)$ are shown. All values are medians. Closed circles with solid lines represent patients infected with mucoid $P$ aeruginosa $(n=9)$, open circles are patients with classic bacteriology $(n=23)$, and crosses patients with cystic fibrosis who died of chronic $P$ aeruginosa infection $(n=12)$. The scales of the ordinates are different from those in figure 1. Dat a from patients with mucoid infection appear in both figures for comparison.
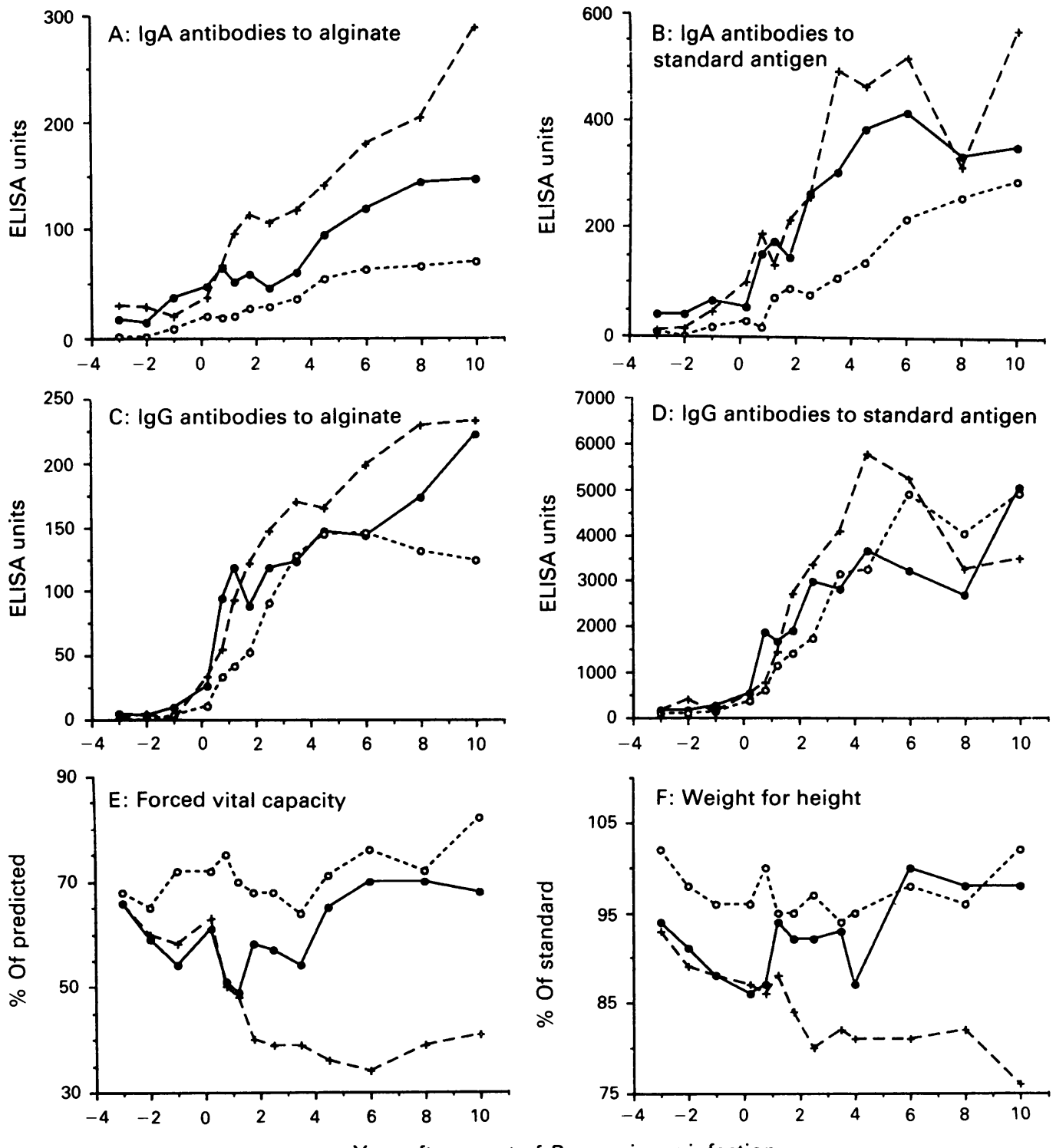


\section{ANTIBODY RESPONSE TO $S$ AUREUS AND $H$} INFLUENZAE

$S$ aureus and $H$ influenzae are common respiratory pathogens and may influence the course of disease. Before the onset of infection those who became infected with mucoid strains had significantly more precipitating antibodies to $S$ aureus than did any of the other groups (data not shown). After onset there were no significant differences in the antibody responses to $S$ aureus and $H$ influenzae between any of the groups. In particular, those who died had a similar antibody response to that of the survivors. The responses remained constant throughout the period of infection and ranged from two to four precipitins (data not shown).

\section{Discussion}

The presence of alginate producing mucoid $P$ aeruginosa is characteristically associated with chronic pulmonary infection in patients with cystic fibrosis. In some cystic fibrosis centres $80 \%$ or more of the patients harbour mucoid strains. ${ }^{3}$ Acquisition of mucoid strains usually leads to a deteriorating clinical state that coincides with systemic humoral hyperimmunisation. ${ }^{1422}$ Evidence has accumulated suggesting that $P$ aeruginosa antibodies contribute to the pathogenicity of progressive lung damage in cystic fibrosis. Tissue damage is thought to be due to inflammation mediated by immune complexes, which cause release of proteases from polymorphonuclear leucocytes. ${ }^{12}{ }^{13}$ Any bacterial product that induces a noticeable antibody response must therefore be regarded as a virulence factor in cystic fibrosis lung disease.

The aim of our study was to test the hypothesis that alginate is a virulence factor in $P$ aeruginosa infection in patients with cystic fibrosis. In most patients the initial infecting strain is non-mucoid, but only a small proportion of strains remain so. In this study it was possible to select a group of patients with stable mucoid or non-mucoid infection throughout 10 years of observation, which allowed the virulence properties of alginate to be assessed. We found that infection with alginate producing mucoid $P$ aeruginosa was associated with a poorer clinical condition, although this does not constitute proof of a causal relation. Evidence in support of alginate being a virulence factor is as follows. The antibody response was greater and the FVC and nutritional state were poorer in patients with mucoid infection compared with patients infected with non-mucoid strains, while nonmucoid strains were associated with a pattern of disease that was similar to that in patients not infected with $P$ aeruginosa. Non-mucoid strains could therefore be considered harmless in cystic fibrosis. ${ }^{6}$ All the patients who died were infected with mucoid strains. The only known bacterial product mucoid strains produce in excess compared with non-mucoid strains is alginate. Mucoid strains must be considered to be deficient organisms because they are serum sensitive and polyagglutinable owing to loss of the lipopolysaccharide side chain and because they produce less toxins than non-mucoid strains. ${ }^{23} 24$ It may be argued that alginate is not a virulence factor because the patients infected with these organisms - that is, those with mucoid and classic bacteriology-were able to maintain lung function and nutritional state virtually unchanged during a long period of infection. The patients in this study, however, do not represent the natural course of infection because they were treated intensively with antibiotics. Furthermore, these patients had survived the infection for about 10 years and constitute a selected group. In addition, they were unable to improve their FVC above preinfection values in contrast to those with non-mucoid infection. A contribution by other bacterial products to the observed differences cannot be ruled out, but we believe that alginate is the most important bacterial factor to determine the course of $P$ aeruginosa lung infection in cystic fibrosis.

Patients who later acquired either mucoid or non-mucoid infection had a greater IgA antibody response than non-colonised patients before infection was apparent. This indicates that $P$ aeruginosa was present, possibly intermittently, and was immunogenic in the lungs up to two years before it was isolated by sputum culture. The absence of $P$ aeruginosa on culture may not necessarily therefore represent absence of $P$ aeruginosa in the lungs; it may be present intermittently or in quantities that are too small to be detected (lower limit $5 \times 10^{2}$ organisms $/ \mathrm{ml}$ sputum). Brett et al showed that specific IgG antibodies became detectable before isolation of the organism, ${ }^{25}$ but we were unable to confirm this. Determination of IgA antibodies, however, may be useful for early detection of infection. Frequent bacteriological examinations, eventually using bronchoalveolar lavage fluid or bigger culture inocula, may be advisable when there is clinical or serological suspicion of $P$ aeruginosa infection.

The data presented confirm our earlier findings that patients with non-mucoid strains produce antibodies to alginate. ${ }^{11}$ The term nonmucoid is therefore misleading if it is assumed to mean that the organism does not produce alginate. The mucoid nature of $P$ aeruginosa is unstable on subsequent culture, ${ }^{26}$ and the bacteria in vivo may exist in the mucoid phenotype. ${ }^{27}$ The genes for alginate biosynthesis are present in non-mucoid strains ${ }^{22} 28$ and a low level of alginate production has been reported for non-mucoid $P$ aeruginosa. ${ }^{29}$ A greater antibody response is associated with the presence of mucoid strains than of non-mucoid strains (figure 1, A-D). This increased antibody response could be because alginate is an adjuvant or a polyclonal $B$ cell activator, though we have found that alginate is not a polyclonal B cell activator (unpublished observations). This is in accordance with our finding that the antibody response to $P$ aeruginosa alone is increased in patients with mucoid infection.

The antibody response in patients with classic bacteriology showed a similar response initially to that seen for non-mucoid strains, changing after three years to the course seen in 
patients with mucoid strains (figure 2). The serological response of patients who died from $P$ aeruginosa infection did not differ from that in patients with mucoid infection. Interestingly, however, the IgA antibody response to standard antigen was significantly greater during the early stage of infection in those who subsequently died than in survivors with the classic pattern of colonisation. The concentration of IgG antibodies did not discriminate between the subsequent progress of disease. Circulating immune complexes are associated with increased IgA concentrations ${ }^{31}$ and high total IgA concentrations occurred in patients who subsequently developed severe pulmonary complications. ${ }^{32}$ Patients who died had higher IgA concentrations than those who survived. ${ }^{33}$ Determination of IgA antibodies may be useful to monitor the progression of lung disease. It is not possible from this study to determine whether increased IgA concentrations are an epiphenomenon of more aggressive infection or have a causal relation to the disease process. IgA coats $P$ aeruginosa in the lungs. ${ }^{34}$ Specific IgA antibodies may promote the persistence of bacteria in the lungs by blocking the attachment of opsonising IgG antibodies, ${ }^{35}$ by inhibiting intracellular bacterial lysis, ${ }^{36}$ or by steric interference with complement fixation. ${ }^{37}$ IgA antibodies may also be more directly involved: acute lung injury has been induced experimentally in rats by $\operatorname{IgA}$ immune complexes. ${ }^{38} 39$

The propensity for $P$ aeruginosa to infect patients with cystic fibrosis is not understood, but despite uniform treatment principles patients who developed chronic $P$ aeruginosa infection had poorer lung function before the onset of infection than those who did not become infected for the next 10-15 years (figures 1E and 2E). This confirms data indicating that pulmonary disease tends to be more advanced in those who later acquire $P$ aerugin$o s a,{ }^{33}$ but contradicts the findings of Kerem $e t$ $a l$, who found no difference in lung function one year before colonisation, although $64 \%$ of their patients acquired $P$ aeruginos $a$ infection before 7 years of age, when cooperation with pulmonary function testing is less reliable. ${ }^{40}$ Our data suggest that significant lung damage had occurred before the onset of $P$ aeruginosa infection, which is in keeping with the observation that $P$ aeruginosa is not usually the first pathogen in the lung in cystic fibrosis. The patients with mucoid infection had a higher antibody response to $S$ aureus in the years preceding the onset of $P$ aeruginosa infection, possibly indicating a predilection of mucoid strains for severely damaged airways. The implication of this finding is that more vigorous treatment of bacterial pathogens-for example, $S$ aureus and $H$ influenzae - and perhaps also viral episodes, which may precede $P$ aeruginosa infection, ${ }^{41}$ may be helpful.

In patients given intensive treatment a large proportion will maintain similar lung function to that before infection, indicating that infection in these patients does not necessarily lead to a progressive decline in lung function as described previously ${ }^{42}$ and supporting earlier reports from our own ${ }^{15}$ and other centres. ${ }^{40}$ Obviously there is a subset of patients whose condition deteriorates and in whom the infection is fatal; further studies are needed to identify the factors responsible for this unfavourable clinical course.

The influence of malabsorption on lung function and the influence of respiratory infection on nutritional state are matters for debate. In our study weight and height were similar in patients with classic bacteriology or nonmucoid infection and in non-colonised patients. There was no difference in treatment other than antipseudomonal chemotherapy between the groups, nutritional advice and pancreatic enzyme supplementation being the same. These findings support the view that failure to thrive in cystic fibrosis relates more closely to chronic pulmonary infection than to pancreatic insufficiency, which can be corrected by enzyme substitution. ${ }^{43}$ Patients in a centre recommending a high energy diet had a better nutritional state and increased survival rates in one study.$^{44}$ We rarely use hyperalimentation and have only recommended a high energy diet for the past five years. Low body weight in cystic fibrosis may be due to increased concentrations of tumour necrosis factor alpha induced by $P$ aeruginosa infection. ${ }^{45}$ Our data show that pulmonary function can be maintained despite chronic $P$ aeruginosa infection and that nutritional states similar to those of children without cystic fibrosis can be established. This is in keeping with our previous results showing that aggressive antipseudomonal chemotherapy is associated with improved survival ${ }^{1546}$ and accords with the strong correlation seen between survival and the use of antibiotics. ${ }^{47} \mathrm{We}$ believe that aggressive antibiotic treatment of lung infections is mandatory in cystic fibrosis.

Our study has shown that the clinical condition of patients with mucoid infection is impaired compared with that of patients with non-mucoid infection and that the condition did not improve with chemotherapy. The importance of alginate in infection is likely to be related to the amount produced as small amounts are produced by non-mucoid strains. The role of alginate in the persistence of chronic infection is well described. We suggest that in addition to its being an antigen its role in the pathogenesis of lung disease is as an immunological adjuvant contributing broadly to increased formation of immune complexes. The genetics of alginate biosynthesis is an area of active research interest and the possibility of engineering a switchback to the non-mucoid phenotype could be immensely important for patients with cystic fibrosis infected with mucoid strains.

We thank Mrs Lena Nørregaard for expert technical help. This study was supported by grants to SSP from the Danish Medical Research Council and the Danish Cystic Fibrosis Association.

1 Wood RE, Boat TF, Doershuk CF. State of the art: cystic fibrosis. Am Rev Respir Dis 1976;113:833-78.

2 di Sant Agnese PA, Davis PB. Cystic fibrosis in adults, 75 cases and a review of 232 cases in the literature. Am J Med 1979;66:121-32.

3 Høiby N. Microbiology of lung infections in cystic fibrosis. 
Acta Paediatr Scand Suppl 1982;301:33-54.

4 Doggett RG, Harrison GM, Stillwell RN, Wallis ES. An atypical Pseudomonas aeruginosa associated with cystic fibrosis of the pancreas. $J$ Pediatr 1966;68:215-21.

5 Høiby N. Pseudomonas aeruginosa infection in cystic fibrosis. Relationship between mucoid strains of Pseudomonas aeruginosa and the humoral immune response. Acta Pathologica et Microbiologica Scandinavica Section B 1974;82:551-8.

6 Henry RL, Dorman DC, Brown J, Mellis C. Mucoid Pseudomonas aeruginosa in cystic fibrosis. Aust Paediatr $J$ 1982;18:43-5.

7 Høiby N, Flensborg EW, Beck B, Friis B, Jacobsen SV, Jacobsen L. Pseudomonas aeruginosa infection in cystic fibrosis. Diagnostic and prognostic significance of Pseudomonas aeruginosa precipitins determined by means of crossed immunoelectrophoresis. Scandinavian Journal of Respiratory Diseases 1977;58:65-79.

8 Demko CA, Byard PJ. Long-term effect of Pseudomonas aeruginosa colonization on pulmonary function in patients with cystic fibrosis (Abstract 74). Pediatr Pulmonol 1988;2(suppl): 115.

9 Burns MW, May JR. Bacterial precipitins in serum of patients with cystic fibrosis. Lancet 1968;i:270-2.

10 Doggett RG, Harrison GM. Pseudomonas aeruginosa: immune status in patients with cystic fibrosis. Infect Immun 1972;6:628-35.

11 Pedersen SS, Espersen F, Høiby N, Jensen T. Immunoglobulin $A$ and immunoglobulin $G$ antibody responses to alginates from Pseudomonas aeruginosa in patients with cystic fibrosis. J Clin Microbiol 1990;28:747-55.

12 Moss RB. Immunology of cystic fibrosis: immunity, immunodeficiency and hypersensitivity. In: Lloyd-Stil JD, ed: Textbook of cystic fibrosis, Boston: John Wright, 1983:109-51.

13 Høiby N, Döring G, Schiøtz PO. The role of immune complexes in the pathogenesis of bacterial infection. Ann Rev Microbiol 1986;40:29-53.

14 Høiby N, Andersen V, Bendixen G. Pseudomonas aeruginosa infection in cystic fibrosis. Humoral and cellular immune response against Pseudomonas aeruginosa infection. Acta Pathologica et Microbiologica Scandinavica Section $C$ 1975;83:459-68.

15 Pedersen SS, Jensen T, Høiby N, Koch C, Flensborg EW. Management of Pseudomonas aeruginosa lung infection in Danish cystic fibrosis patients. Acta Paediatr Scand 1987;76:955-61.

$16 \mathrm{Jessen} \mathrm{O}$. Pseudomonas aeruginosa and other green fluorescent pseudomonads. Copenhagen: Munksgaard, 1965:83-97.

17 Polgar GE, Promadhat V. Pulmonary function testing in children: techniques and standards. Philadelphia: WB Saunders, 1971:88-107.

18 Andersen E, Hutchings B, Jansen J, Nyholm M. Højde og vaegt hos danske born. Ugeskr Laeger 1982;144:1760-5.

19 Høiby N. Pseudomonas aeruginosa infection in cystic fibrosis. Diagnostic and prognostic significance of Pseudomonas aeruginosa precipitins determined by means of crossed immunoelectrophoresis: a survey. Acta Pathologica Microbiologica Scandinavica Section C;262(suppl)3-96.

20 Pedersen SS, Espersen F, Høiby N, Shand GH. Purification, characterization and immunological cross-reactivity of alginates produced by mucoid Pseudomonas aeruginosa from patients with cystic fibrosis. J Clin Microbiol 1989;27:691-9.

21 Pedersen SS, Espersen F, Høiby N. Diagnosis of chronic Pseudomonas aeruginosa infection in cystic fibrosis by Pseudomonas aeruginosa infection in cystic fibrosis by 1987;25:1830-6.

22 Govan JRW. Alginate biosynthesis and other unusual characteristics associated with the pathogenesis of Pseudomonas aeruginosa in cystic fibrosis. In: Griffiths $\mathrm{E}$ Donachie W, Stephen J, eds. Bacterial infections of respiratory and gastrointestinal mucosae. Oxford: IRL Press, 1988:67-96.

23 Ohman DE, Chakrabarty AM. Utilization of human respiratory secretions by mucoid Pseudomonas aeruginosa of cystic fibrosis origin. Infect Immun 1982;37:662-9.

24 Woods DE, Schaffer MS, Rabin HR, Campbell GD, Sokol PA. Phenotypic comparison of Pseudomonas aeruginosa strains isolated from a variety of clinical sites. J Clin Microbiol 1986;24:260-4

25 Brett MM, Ghoneim ATM, Littlewood JM. Prediction and diagnosis of early Pseudomonas aeruginosa infection in cystic fibrosis: a follow-up study. J Clin Microbiol cystic fibrosis:

26 Zierdt CH, Williams RL. Serotyping of Pseudomonas aeruginosa isolated from patients with cystic fibrosis of the pancreas. J Clin Microbiol 1975;1:521-6.

27 Thomassen MI, Demko CA, Boxerbaum B, Stern RC Kuchenbrod PJ. Multiple isolates of Pseudomonas aeruginosa with differing antimicrobial susceptibility patterns from patients with cystic fibrosis. I Infect Dis 1979;140:873-80.

28 Sá-Correa I, Darzins A, Wang S-K, Berry A, Chakrabarty AM. Alginate biosynthetic enzymes in mucoid and nonmucoid Pseudomonas aeruginosa: overproduction of phosphomannose isomerase, phosphomannomutase, and GDP-mannose pyrophosphorylase by overexpression of the phosphomannose isomerase (pmi) gene. J Bacteriol the phosphomann

29 Pier GB, Desjardins D, Aguilhar T, Barnard M, Speert DP. Polysaccharide surface antigens expressed by nonmucoid isolates of Pseudomonas aeruginosa from cystic fibrosis patients. J Clin Microbiol 1986;24:189-96.

30 Anastassiou ED, Mintzas AC, Kounavis C, Dimitracopoulos G. Alginate production by clinical nonmucoid Pseudomonas aeruginosa strains. J Clin Microbiol 1987;25:656-9.

31 Moss RB, Lewiston NJ. Immune complexes and humoral response to Pseudomonas aeruginosa in cystic fibrosis. $\mathrm{Am}$ Rev Respir Dis 1980;121:23-9.

32 Schwartz RH. Serum immunoglobulin levels in cystic fibrosis. Am J Dis Child 1966;111:408-11.

33 Wisnieski JJ, Todd EW, Fuller RK et al. Immune complexes and complement abnormalities in patients with plexes and complement abnormalities in patients with ing immune complexes and decreased function of the ing immune complexes and decreased function of the alternative comple

34 Speert DP, Dimmick JE, Pier GB, Saunders JM, Hancock REW, Kelly N. An immunohistological evaluation of Pseudomonas aeruginosa pulmonary infection in two patients with cystic fibrosis. Pediatr Res 1987;18:431-3.

35 Musher DM, Goree A, Baughn RE, Birdsall HH. Immunoglobulin A from bronchopulmonary secretions blocks bactericidal and opsonizing effects of antibody to nontypeable Haemophilus influenzae. Infect Immun 1984;45:36-40.

36 Piedra P, Ogra PL. Immunologic aspects of surface infections in the lung. J Pediatr 1986;108:817-23.

37 Russell-Jones GJ, Ey PL, Reynolds BL. The ability of IgA to inhibit the complement-mediated lysis of target red to inhibit the complement-mediated lysis of target red
blood cells sensitized with IgG antibody. Mol Immunol 1980;17:1173-80.

38 Johnson KJ, Wilson BS, Till GO, Ward PA. Acute lung injury in rat caused by immunoglobulin $A$ immune complexes. $J$

39 Warren JS, Kunkel RG, Johnson KJ, Ward PA. Comparative $\mathrm{O}_{2}^{-}$responses of lung macrophages and blood phagocytic cells in the rat. Possible relevance of IgA immune complex induced lung injury. Lab Invest 1987;57:311-20.

40 Kerem E, Corey M, Gold R, Levison H. Pulmonary function and clinical course in patients with cystic fibrosis after pulmonary colonization with Pseudomonas aeruginosa. J Pediatr 1990;116:714-9.

41 Petersen NT, Høiby N, Mordhorst CH, Lind K, Flensborg EW, Bruun B. Respiratory infections in cystic fibrosis patients caused by virus, chlamydia and mycoplasmapossible synergism with Pseudomonas aeruginosa. Acta Paediatr Scand 1981;70:623-8.

42 Corey $M$, Levison $H$, Crozier D. Five- to seven-year course of pulmonary function in cystic fibrosis. Am Rev Respir Dis

43 Anonymous. Supplementary nutrition in cytic fibrosis. [Editorial.] Lancet 1986; i:249-51.

44 Corey M, McLaughlin FL, Williams M, Levison H. A comparison of survival, growth, and pulmonary function in patients with cystic fibrosis in Boston and Toronto. $J$ Clin Epidemiol 1988;41:585-91.

45 Suter S, Schaad UB, Roux-Lombard P, Girardin E, Grau G, Dayer J-M. Relation between tumor necrosis factoralpha and granulocyte elastase- $\alpha 1$-proteinase inhibitor complexes in the plasma of patients with cystic fibrosis. $\mathrm{Am}$ Rev Respir Dis 1989;140:1640-4.

46 Szaff $M$, Hoiby N, Flensborg EW. Frequent antibiotic therapy improves survival of cystic fibrosis patients with therapy improves survival of cystic fibrosis patients with Scand 1983;72:651-7.

47 Wood RE, Piazza F. Survival in cystic fibrosis: correlation with treatment in three cystic fibrosis centres. Abstract $\mathbf{R}(0) 1$, 10th International Cystic Fibrosis Congress, Excerpta Medica Asia Pacific Congress Series 74, p. 79. 